\title{
Some criteria for determining when a Walsh Series is a Walsh-Fourier Series
}

\author{
J. Alexopoulos*and E. H. Sprague ${ }^{\dagger}$
}

September 5, 2002

\begin{abstract}
We show that a general Walsh series is the Walsh-Fourier series of a function $f \in L_{p}[0,1]$ for $1 \leq p<\infty$ if and only if its sequence of partial sums contains a relatively weakly compact subsequence. Several other criteria are established for the case where $f \in L_{\Phi}[0,1]$, the Orlicz space generated by an $N$-function $\Phi$.
\end{abstract}

Mathematics subject classification (2000): 42C10, 46E30

Key Words: Walsh series, Orlicz spaces

\section{Introduction and background}

A characterization of the Trigonometric-Fourier series of certain functions in $L_{1}[0,2 \pi]$, by way of the classical theorem of De La Vallée Poussin stated below, is studied in Chapter IV.5 of A. Zygmund's Trigonometric Series [10, p. 145]. This discussion led us to consider whether we can know when an infinite Walsh series is the Walsh-Fourier series for a function in $L_{p}[0,1], 1 \leq p<\infty$ or, in fact, $L_{\Phi}[0,1]$ for some $N$-function $\Phi$. There are many deep results in the literature addressing these types of questions (for example [8, chapter 7]).

A key notion in this paper is that of uniform integrability: Recall that a subset $\mathcal{K}$ of $L_{1}[0,1]$ is called uniformly integrable if $\lim _{c \rightarrow \infty} \sup \left\{\int_{[|f| \geq c]}|f|: f \in \mathcal{K}\right\}=0$. Alternatively, $\mathcal{K} \subseteq L_{1}[0,1]$ is uniformly integrable if and only if it is $L_{1}$-bounded and for each $\varepsilon>0$ there is a $\delta>0$ so that $\sup \left\{\int_{A}|f|: f \in \mathcal{K}\right\}<\varepsilon$ for all measurable $A$ with $\lambda(A)<\delta$ ( $\lambda$ denotes Lebesgue measure on $[0,1])$. The theorem of Dunford and Pettis (see

\footnotetext{
${ }^{*}$ The first author would like to express his gratitude for the financial support and the opportunity to lecture on this material at Universidad Central de Venezuela, Caracas-Venezuela and Universidad de Los Andes, Merida-Venezuela, during the summer of 2002 .

†Portions of these results, and the motivation behind them, were presented in sessions of the Measure Theory and the Non-Linear Analysis seminars at Kent State University, Kent, Ohio during the academic years 2000-2001 and 2001-2002.
} 
$\left[3\right.$, p. 93]) which states that a subset $\mathcal{K}$ of $L_{1}[0,1]$ is uniformly integrable if and only if it is relatively weakly compact, is a well known characterization of uniformly integrable subsets of $L_{1}[0,1]$. Another characterization of uniform integrability is given by a theorem of De La Vallée Poussin (see [7, p. 19]) which states that $a$ subset $\mathcal{K}$ of $L_{1}[0,1]$ is uniformly integrable if and only if there is a non-negative, convex function $Q$ with $\lim _{t \rightarrow \infty} \frac{Q(t)}{t}=\infty$ so that $\sup \left\{\int_{0}^{1} Q(|f|): f \in \mathcal{K}\right\}<\infty$.

In section 2 we show that a general Walsh series $\left\langle S_{n}\right\rangle_{n \in \mathbf{N}}=\sum_{n=0}^{\infty} a_{n} w_{n}$ is the Walsh-Fourier series of an $f \in L_{1}[0,1]$ if and only if there is a uniformly integrable subsequence $\left\langle S_{n_{k}}\right\rangle_{k \in \mathbf{N}}$ of $\left\langle S_{n}\right\rangle_{n \in \mathbf{N}}$ (theorem 2.1). For $1<p<\infty$ we establish the fact that a general Walsh series $\left\langle S_{n}\right\rangle_{n \in \mathbf{N}}=\sum_{n=0}^{\infty} a_{n} w_{n}$ is a Walsh-Fourier series of an $f \in L_{p}[0,1]$ if and only if there is a subsequence of its partial sums $\left\langle S_{n_{k}}\right\rangle_{k \in \mathbf{N}}$ with bounded $L_{p}$ norms (theorem 2.2).

In section 3 we establish a number of different criteria to determine when a general Walsh series is the Walsh-Fourier series of a function in an Orlicz space. In theorem 3.1 we establish that a general Walsh series $\left\langle S_{n}\right\rangle_{n \in \mathbf{N}}=\sum_{n=0}^{\infty} a_{n} w_{n}$ is a Walsh-Fourier series of an $f \in L_{\Phi}[0,1]$ for some $N$-function $\Phi$ if and only if there is a subsequence of its partial sums $\left\langle S_{n_{k}}\right\rangle_{k \in \mathbf{N}}$ with bounded $L_{\Phi}$ norms while in corollary 3.2 we investigate the special case of $\Phi \in \Delta_{2}$. Subsequently, in corollary 3.3 we use the theorem of De La Vallée Poussin to give additional criteria to determine when a general Walsh series is the Walsh-Fourier series of a function in $L_{1}[0,1]$. Finally in corollary 3.6 we investigate some aspects of the Walsh-Fourier series of a function in an Orlicz space, whose generating function does not satisfy the $\Delta_{2}$ condition.

\subsection{Some facts from the theory of Walsh functions}

We describe the Walsh functions in the Paley ordering [8, p.1].

Let $\left\langle r_{n}\right\rangle_{n \in \mathbf{N}}$ denote the sequence of Rademacher functions. That is, let

$$
r_{0}(x)=\left\{\begin{array}{rl}
1 & \text { if } x \in\left[0, \frac{1}{2}\right) \\
-1 & \text { if } x \in\left[\frac{1}{2}, 1\right)
\end{array} .\right.
$$

We extend $r_{0}$ to the whole real line periodically with period one and define

$$
r_{n}(x)=r_{0}\left(2^{n} x\right), \text { for } n \in \mathbf{N}
$$

For $m \in \mathbf{N}$ we find the $m^{\text {th }}$ Walsh function, $w_{m}:[0,1) \rightarrow\{-1,1\}$, in the Paley ordering. For $m=0$ define

$$
w_{0}(x)=1, \quad \forall x \in[0,1]
$$

For $m>0$ we write the dyadic expansion of $m$ :

$$
m=\sum_{k=0}^{\infty} e_{k} 2^{k}, e_{k} \in\{0,1\}
$$


and define

$$
w_{m}(x)=\prod_{k=0}^{\infty}\left(r_{k}(x)\right)^{e_{k}}
$$

where $e_{k}$ is the $k^{\text {th }}$ dyadic coefficient of $m$. Since all but finitely many of the $e_{k}$ 's are zero, the Walsh functions in the Paley ordering are finite products of the $r_{n}$ 's It turns out that there is a correspondence between the Walsh functions in the Paley ordering and the collection of continuous characters on the Dyadic Group. From this isomorphism, we find that the Walsh functions in the Paley ordering form an orthonormal basis in $L_{2}[0,1]$. ([8, Chapter 1$\left.]\right)$

1. Definition: For $x \in[0,1]$ we write the dyadic expansion of $x, x=\sum_{n=0}^{\infty} x_{n} 2^{-(n+1)}$. For the dyadic rationals, we choose the sum whose final terms are zero. We define dyadic addition on $[0,1) \times[0,1)$, $+_{D}$, by

$$
x+{ }_{D} y=\sum_{n=0}^{\infty}\left|x_{n}-y_{n}\right| 2^{-(n+1)}([8, \text { p. } 10])
$$

2. Paley's Lemma: $\sum_{k=0}^{2^{n}-1} w_{k}(x)=\left\{\begin{array}{l}2^{n} \text { if } x \in\left[0, \frac{1}{2^{n}}\right) \\ 0 \text { otherwise }\end{array} \quad([8\right.$, p. 7$])$

3. Lemma: If $x$ and $y$ are in the same $n^{\text {th }}$ dyadic interval, $\left[\frac{j}{2^{n}}, \frac{j+1}{2^{n}}\right)$, then $x+_{D} y$ is in $\left[0, \frac{1}{2^{n}}\right)$. This follows from the fact that when $x$ and $y$ are in the same dyadic interval, the first $n$ terms of the dyadic sums of $x$ and $y$ are equal, so $\left|x_{k}-y_{k}\right|=0$ for $k \leq n$.

4. Lemma: $w_{n}(x) w_{n}(y)=w_{n}\left(x+_{D} y\right)([8$, p. 10] $)$

5. Notation: For the rest of this paper

- $S=\sum_{n \in \mathbf{N}} a_{n} w_{n}$ is an arbitrary Walsh series with Real coefficients.

- $S f=\sum_{n \in \mathbf{N}} \hat{f}_{n} w_{n}$ is a Walsh-Fourier series of a function $f \in L_{0}[0,1]$, where the $n^{\text {th }}$ coefficient of the series is $\hat{f}_{n}=\int_{0}^{1} f(t) w_{n}(t) d t$.

- $S_{n}=\sum_{k=0}^{n-1} a_{n} w_{k}$ is the $n^{\text {th }}$ partial sum of a Walsh series. $S_{n} f=\sum_{k=0}^{n-1} \hat{f}_{k} w_{k}$ is the $n^{\text {th }}$ partial sum of a Walsh-Fourier series.

\subsection{Some facts from the theory of Orlicz Spaces}

We recall some basic facts about $N$-functions and Orlicz Spaces. For a detailed account of these facts, the reader could consult chapters one and two in [6].

1. Definition: A function $\Phi$ is an $N$-function if and only if $\Phi$ is continuous, even and convex with

(a) $\lim _{x \rightarrow 0} \frac{\Phi(x)}{x}=0$; 
(b) $\lim _{x \rightarrow \infty} \frac{\Phi(x)}{x}=\infty$;

(c) $\Phi(x)>0$ if $x>0$.

2. Definition: For an $N$-function $\Phi$ define $\Psi(x)=\sup \{t|x|-\Phi(t): t \geq 0\}$. Then $\Psi$ is an $N$-function and it is called the complement of $\Phi$.

Observe that $\Phi$ is the complement of its complement $\Psi$. Given an $N$-function $\Phi$, the corresponding space of $\Phi$-integrable functions is defined as follows:

3. Definition: For an $N$-function $\Phi$ and a measurable $f$ define

$$
\boldsymbol{\Phi}(f)=\int_{0}^{1} \Phi(f) .
$$

If $\Psi$ denotes the complement of $\Phi$ let

$$
L_{\Phi}=\left\{f \text { measurable }:\left|\int_{0}^{1} f g\right|<\infty, \forall g \text { with } \mathbf{\Psi}(g)<\infty\right\}
$$

The collection $L_{\Phi}$ is then a linear space. For $f \in L_{\Phi}$ define

$$
\|f\|_{\Phi}=\sup \left\{\left|\int_{0}^{1} f g\right|: \Psi(g) \leq 1\right\}
$$

Then $\left(L_{\Phi},\|\cdot\|_{\Phi}\right)$ is a Banach space, called an Orlicz space. Moreover, letting $\|f\|_{(\Phi)}=\inf \left\{k>0: \Phi\left(\frac{f}{k}\right) \leq 1\right\}$ be the Minkowski functional associated with the convex set $\left\{f \in L_{\Phi}: \Phi(f) \leq 1\right\}$, we have that $\|\cdot\|_{(\Phi)}$ is an equivalent norm on $L_{\Phi}$, called the Luxemburg norm. Indeed, $\|f\|_{(\Phi)} \leq\|f\|_{\Phi} \leq 2\|f\|_{(\Phi)}$, for all $f \in L_{\Phi}$.

4. Theorem: Let $\Phi$ be an $N$-function and let $E_{\Phi}$ be the closure of the bounded functions in $L_{\Phi}$. Then the conjugate space of $\left(E_{\Phi},\|\cdot\|_{(\Phi)}\right)$ is $\left(L_{\Psi},\|\cdot\|_{\Psi}\right)$, where $\Psi$ is the complement of $\Phi$.

5. Definition: An $N$-function $\Phi$ is said to satisfy the $\Delta_{2}$ condition $\left(\Phi \in \Delta_{2}\right)$ if $\limsup _{x \rightarrow \infty} \frac{\Phi(2 x)}{\Phi(x)}<\infty$. That is, there is a $K>0$ so that $\Phi(2 x) \leq K \Phi(x)$ for large values of $x$.

6. Definition: We say that a collection $\mathcal{K} \subset L_{\Phi}$ has equi-absolutely continuous norms if and only if it is norm bounded and $\forall \varepsilon>0 \exists \delta>0$ so that $\sup \left\{\left\|\chi_{E} f\right\|_{\Phi}: f \in \mathcal{K}\right\}<\varepsilon$ whenever $\lambda(E)<\delta$.

7. Theorem: Let $\Phi$ be an $N$-function and $\Psi$ be its complement. Then the following statements are equivalent:

(a) $L_{\Phi}=E_{\Phi}$.

(b) $L_{\Phi}=\{f$ measurable : $\mathbf{\Phi}(f)<\infty\}$.

(c) The dual of $\left(L_{\Phi},\|\cdot\|_{(\Phi)}\right)$ is $\left(L_{\Psi},\|\cdot\|_{\Psi}\right)$. 
(d) $\forall f \in L_{\Phi},\{f\}$ has equi-absolutely continuous norms.

(e) $\Phi \in \Delta_{2}$.

8. In [1] J. Alexopoulos has shown that

(a) Theorem: If $\mathcal{K} \subseteq L_{\Phi}$ has equi-absolutely continuous norms, then $\mathcal{K}$ is a Banach-Saks set in $L_{\Phi}$. In particular $\mathcal{K}$ is relatively weakly compact in $L_{\Phi}([1$, thm. 2.3$])$.

(b) Theorem: A set $\mathcal{K}$ is relatively weakly compact in $L_{1}$ if and only if there is $\Phi \in \Delta_{2}$ so that $\mathcal{K}$ has equi-absolutely continuous norms (in $\left.L_{\Phi}\right)$ and it is thus relatively weakly compact in $L_{\Phi}([1$, thm. 2.5]).

(c) If $\Phi \in \Delta_{2}$ and $\mathcal{K} \subset L_{\Phi}$ then the following statements are equivalent:

i. The set $\mathcal{K}$ has equi-absolutely continuous norms.

ii. The collection $\{\Phi(f): f \in \mathcal{K}\}$ is uniformly integrable.

\section{The case of $L_{p}[0,1]$ for $1 \leq p<\infty$}

Our first theorem characterizes those Walsh series which are Walsh-Fourier series of a function $f \in L_{1}[0,1]$ :

Theorem 2.1 A general Walsh series $\left\langle S_{n}\right\rangle_{n \in \mathbf{N}}=\sum_{n=0}^{\infty} a_{n} w_{n}$ is the Walsh-Fourier series of an $f \in L_{1}[0,1]$ if and only if there is a uniformly integrable subsequence $\left\langle S_{n_{k}}\right\rangle_{k \in \mathbf{N}}$ of $\left\langle S_{n}\right\rangle_{n \in \mathbf{N}}$.

Proof: In order to prove sufficiency it is enough to recall that $S_{2^{n}} f \rightarrow f$ in $L_{1}$ norm ([8, p. 142]) and thus $\left\langle S_{2^{n}} f\right\rangle_{n \in \mathbf{N}}$ is relatively norm-compact. Hence $\left\langle S_{2^{n}}\right\rangle_{n \in \mathbf{N}}$ is relatively weakly-compact and consequently uniformly integrable, thanks to the Dunford-Pettis theorem. An alternative, insightful proof relies on a famous theorem of Doob [2, p. 336]:

Doob's martingale convergence theorem: If $\left\langle f_{n}, \mathbf{A}_{n}\right\rangle_{n \in \mathbf{N}}$ is a martingale, the following are equivalent:

1. $\left\langle f_{n}\right\rangle_{n \in \mathbf{N}}$ is a uniformly integrable sequence.

2. $\left\langle f_{n}\right\rangle_{n \in \mathbf{N}}$ converges in $L_{1}$.

3. There exists an integrable $g$ so that $f_{n}=E\left(g \mid \mathbf{A}_{n}\right) \forall n \in \mathbf{N}$

In particular, if any of (1), (2) and (3) are satisfied then there is a function $g \in L_{1}$ such that $f_{n} \rightarrow g$ almost surely.

With this theorem in hand, we proceed as follows: 
For any $n$, let $\mathbf{A}_{n}$ be the $\sigma$-algebra of subsets of $[0,1]$ generated by the collection of the $2^{n^{\text {th }}}$ dyadic intervals, $\left\{\left[\frac{k}{2^{n}}, \frac{k+1}{2^{n}}\right), 0 \leq k<2^{n}\right\}$. The sequence $\left\langle S_{2^{n}}, \mathbf{A}_{n}\right\rangle_{n \in \mathbf{N}}$, is a martingale. ([8, p. 75]) We assume that $a_{n}=$ $\hat{f}_{n}, \forall n$, for some $f \in L_{1}[0,1]$ and we show that $\forall n \in \mathbf{N}, S_{2^{n}} f=\mathbf{E}\left(f \mid \mathbf{A}_{n}\right),\left(\mathbf{A}_{n}\right)$-almost surely:

Fix $n \in \mathbf{N}$ and $0 \leq j<2^{n}$. Then

$$
\begin{aligned}
\int_{\frac{j}{2^{n}}}^{\frac{j+1}{2^{n}}}\left(S_{2^{n}} f\right)(x) d x & =\int_{\frac{j}{2^{n}}}^{\frac{j+1}{2^{n}}} \sum_{k=0}^{2^{n}-1}\left(\int_{0}^{1} f(t) w_{k}(t) d t\right) w_{k}(x) d x \\
& =\int_{\frac{j}{2^{n}}}^{\frac{j+1}{2^{n}}} \int_{0}^{1} f(t)\left(\sum_{k=0}^{2^{n}-1} w_{k}\left(x+{ }_{D} t\right)\right) d t d x \\
& =\int_{\frac{j}{2^{n}}}^{\frac{j+1}{2^{n}}}\left(\int_{[0,1) \backslash\left[\frac{j}{2^{n}}, \frac{j+1}{2^{n}}\right]} f(t) \sum_{k=0}^{2^{n}-1} w_{k}\left(x+{ }_{D} t\right) d t+\int_{\frac{j}{2^{n}}}^{\frac{j+1}{2^{n}}} f(t) \sum_{k=0}^{2^{n}-1} w_{k}\left(x+{ }_{D} t\right) d t\right) d x \\
& =\int_{\frac{j}{2^{n}}}^{\frac{j+1}{2^{n}}}\left(0+2^{n} \int_{\frac{j}{2^{n}}}^{\frac{j+1}{2^{n}}} f(t) d t\right) d x \quad(\text { by } 1.1 \text { note } 3 \text { and the Paley Lemma) } \\
& =\int_{\frac{j}{2^{n}}}^{\frac{j}{n}} f(t) d t
\end{aligned}
$$

Hence, by the definition of $\mathbf{A}_{n}, \mathbf{E}\left(f \mid \mathbf{A}_{n}\right)=S_{2^{n}} f, \quad\left(\mathbf{A}_{n}\right)$-almost surely. So, the third condition of Doob's theorem is satisfied and $\left\langle S_{2^{n}} f\right\rangle_{n \in \mathbf{N}}$ is uniformly integrable.

In order to establish necessity, we suppose that there is a uniformly integrable subsequence $\left\langle S_{n_{k}}\right\rangle_{k \in \mathbf{N}}$ of the sequence of partial sums $\left\langle S_{n}\right\rangle_{n \in \mathbb{N}}$ of the general Walsh series $\sum_{n=0}^{\infty} a_{n} w_{n}$. The Dunford-Pettis Theorem tells us that $\left\langle S_{n_{k}}\right\rangle_{k \in \mathbf{N}}$ is relatively weakly compact and so by the Eberlein-Smullian theorem, there is a weakly convergent subsequence $\left\langle S_{n_{k_{i}}}\right\rangle_{i \in \mathbf{N}}$ of $\left\langle S_{n_{k}}\right\rangle_{k \in \mathbb{N}}$. That is, there is some $f \in L_{1}[0,1]$ such that

$$
\sum_{j=0}^{n_{k_{i}}-1} a_{j} w_{j}=S_{n_{k_{i}}} \stackrel{\text { weakly }}{\longrightarrow} f \text { as } i \rightarrow \infty .
$$

We fix $m \geq 0$ and investigate the behaviour of $\left\langle S_{n_{k_{i}}}\right\rangle_{i \in \mathbf{N}}$.

Noting that for each $m \in \mathbf{N}, w_{m} \in L_{\infty}[0,1]=L_{1}^{*}[0,1]$, we see that

$$
\int_{0}^{1} S_{n_{k_{i}}} w_{m} \rightarrow \int_{0}^{1} f w_{m} \text { as } i \rightarrow \infty .
$$

Now for $n_{k_{i}}>m$, the orthonormality of the Walsh functions yields

$$
\int_{0}^{1} S_{n_{k_{i}}} w_{m}=\int_{0}^{1}\left(\sum_{j=0}^{n_{k_{i}}-1} a_{j} w_{j}\right) w_{m}=\sum_{j=0}^{n_{k_{i}}-1}\left(a_{j} \int_{0}^{1} w_{j} w_{m}\right)=\int_{0}^{1} a_{m} w_{m}^{2}=a_{m} .
$$

Thus

$$
\int_{0}^{1} S_{n_{k_{i}}} w_{m} \rightarrow a_{m} \text { as } i \rightarrow \infty \text { which forces } a_{m}=\int_{0}^{1} f w_{m}=\hat{f}_{n}
$$

and the proof is complete.

We turn now to the case of the Walsh-Fourier series of an $f \in L_{p}$ for some $p>1$ : 
Theorem 2.2 A general Walsh series $\left\langle S_{n}\right\rangle_{n \in \mathbf{N}}=\sum_{n=0}^{\infty} a_{n} w_{n}$ is a Walsh-Fourier series of an $f \in L_{p}[0,1]$ for some $1<p<\infty$, if and only if its sequence of partial sums, $\left\langle S_{n}\right\rangle_{n \in \mathbf{N}}$, contains a subsequence, $\left\langle S_{n_{k}}\right\rangle_{k \in \mathbf{N}}$, with bounded $L_{p}$ norms.

Proof: First we note that given a finite $p>1$ and an $f \in L_{p}[0,1]$, the full sequence of partial sums of the Walsh-Fourier series of $f,\left\langle S_{n} f\right\rangle_{n \in \mathbf{N}}$, converges in $L_{p}$ norm to $f([8, \mathrm{p} .142])$ and thus is $L_{p}$-bounded.

In order to establish the converse, we suppose that there is a $1<p<\infty$ and an $M>0$ such that $\int_{0}^{1}\left|S_{n_{k}}\right|^{p} \leq M$, for all $k \in \mathbf{N}$. Since $\left\langle S_{n_{k}}\right\rangle_{k \in \mathbf{N}}$ is norm bounded in $L_{p}[0,1],\left\langle S_{n_{k}}\right\rangle_{k \in \mathbf{N}}$ is relatively weakly compact in the reflexive Banach space $L_{p}[0,1]([4$, p. 68]), and so by the Eberlein-Smullian theorem again, there must be a subsequence $\left\langle n_{k_{j}}\right\rangle_{j \in \mathbf{N}} \subseteq\left\langle n_{k}\right\rangle_{k \in \mathbf{N}}$ and an $f \in L_{p}[0,1]$ such that $S_{n_{k_{j}}} \stackrel{\text { weakly }}{\rightarrow} f$ as $j \rightarrow \infty$. Now $w_{n} \in L_{q}[0,1]=L_{p}^{*}[0,1]$ (where $\left.\frac{1}{p}+\frac{1}{q}=1\right)$, and so

$$
\int_{0}^{1} S_{n_{k_{j}}} w_{n} \rightarrow \int_{0}^{1} f w_{n} \text { as } j \rightarrow \infty
$$

An argument similar to the one in the previous theorem establishes that,

$$
\int_{0}^{1} S_{n_{k_{j}}} w_{n} \rightarrow a_{n} \text { as } j \rightarrow \infty
$$

and so $a_{n}=\hat{f}_{n}$ as we wanted.

\section{Walsh-Fourier series in Orlicz spaces}

Theorem 3.1 Let $\Phi$ be an $N$-function and $S=\left\langle S_{n}\right\rangle_{n \in \mathbf{N}}=\sum_{n=0}^{\infty} a_{n} w_{n}$ a general Walsh series. The following are equivalent:

1. There is an $f \in L_{\Phi}[0,1]$ so that $a_{n}=\hat{f}_{n}$ for all $n$. That is, $S=S f$ for some $f \in L_{\Phi}[0,1]$.

2. There is a constant $M>0$ and a subsequence $\left\langle S_{n_{k}}\right\rangle_{k \in \mathbf{N}}$ of $\left\langle S_{n}\right\rangle_{n \in \mathbf{N}}$ so that $\left\langle\Phi\left(\frac{1}{M} S_{n_{k}}\right)\right\rangle_{k \in \mathbf{N}}$ is uniformly integrable.

3. There is a constant $M>0$ and a subsequence $\left\langle S_{n_{k}}\right\rangle_{k \in \mathbf{N}}$ of $\left\langle S_{n}\right\rangle_{n \in \mathbf{N}}$ so that $\left\langle\Phi\left(\frac{1}{M} S_{n_{k}}\right)\right\rangle_{k \in \mathbf{N}}$ is $L_{1^{-}}$bounded.

4. There is a subsequence $\left\langle S_{n_{k}}\right\rangle_{k \in \mathbf{N}}$ of $\left\langle S_{n}\right\rangle_{n \in \mathbf{N}}$ so that $\left\langle S_{n_{k}}\right\rangle_{k \in \mathbf{N}}$ is $L_{\Phi}$-bounded.

Proof: $\quad(1 \Rightarrow 2)$ : Suppose there is an $f \in L_{\Phi}[0,1]$ so that $a_{n}=\hat{f}_{n}$ for all $n$. Then, there is a constant $M>0$ so that $\Phi\left(\frac{f}{M}\right) \in L_{1}[0,1]$. By the conditional Jensen inequality we have that

$$
\Phi\left(\frac{1}{M} S_{2^{k}} f\right)=\Phi\left(S_{2^{k}} \frac{f}{M}\right)=\Phi\left(\mathbf{E}\left(\frac{f}{M} \mid \mathbf{A}_{k}\right)\right) \leq \mathbf{E}\left(\Phi\left(\frac{f}{M}\right) \mid \mathbf{A}_{k}\right)=S_{2^{k}} \Phi\left(\frac{f}{M}\right)
$$


and as $\Phi\left(\frac{f}{M}\right) \in L_{1}[0,1]$ Theorem 2.1 ensures that $\left\langle S_{2^{k}} \Phi\left(\frac{f}{M}\right)\right\rangle_{k \in \mathbf{N}}$ and thus $\left\langle\Phi\left(\frac{1}{M} S_{2^{k}} f\right)\right\rangle_{k \in \mathbf{N}}$ are uniformly integrable.

$(2 \Rightarrow 3)$ : Trivial.

$(3 \Rightarrow 4)$ : Suppose that there is a constant $M>0$ and a subsequence $\left\langle S_{n_{k}}\right\rangle_{k \in \mathbf{N}}$ of the partial sums so that $\left\langle\Phi\left(\frac{1}{M} S_{n_{k}}\right)\right\rangle_{k \in \mathbf{N}}$ is $L_{1}$-bounded. Thus, there is a constant $C>1$ so that $\sup _{k \in \mathbf{N}} \int_{0}^{1} \Phi\left(\frac{1}{M} S_{n_{k}}\right) \leq C$. Hence, by the convexity of $\Phi$, for each $k$ we have

$$
\int_{0}^{1} \Phi\left(\frac{1}{M C} S_{n_{k}}\right) \leq \frac{1}{C} \int_{0}^{1} \Phi\left(\frac{1}{M} S_{n_{k}}\right) \leq 1
$$

and so $\left\|S_{n_{k}}\right\|_{(\Phi)} \leq M C$. Therefore $\sup _{k \in \mathbf{N}}\left\|S_{n_{k}}\right\|_{(\Phi)} \leq M C$.

$(4 \Rightarrow 1)$ : Suppose that there is an $L_{\Phi}$-bounded subsequence $\left\langle S_{n_{k}}\right\rangle_{k \in \mathbf{N}}$ of the partial sums of the general Walsh series $\sum_{n=0}^{\infty} a_{n} w_{n}$. Then there is a constant $M>1$ so that $\sup _{k \in \mathbf{N}}\left\|S_{n_{k}}\right\|_{(\Phi)} \leq M$. So $\sup _{k \in \mathbf{N}} \int_{0}^{1} \Phi\left(\frac{S_{n_{k}}}{M}\right) \leq$ 1 and by the theorem of De La Vallée Poussin,

$$
\left\langle\frac{S_{n_{k}}}{M}\right\rangle_{k \in \mathbf{N}} \text { and along with it }\left\langle S_{n_{k}}\right\rangle_{k \in \mathbf{N}}
$$

are uniformly integrable.

By the Dunford-Pettis and Eberlein-Smullian theorems, there is a subsequence $\left\langle S_{n_{k_{j}}}\right\rangle_{j \in \mathbf{N}}$ of $\left\langle S_{n_{k}}\right\rangle_{k \in \mathbf{N}}$ and a function $f \in L_{1}[0,1]$ so that $S_{n_{k_{j}}} \stackrel{L_{1} \text {-weakly }}{\longrightarrow} f$ as $j \longrightarrow \infty$. For each $n, w_{n} \in L_{\infty}[0,1]=L_{1}^{*}[0,1]$ and so $\int_{0}^{1} w_{n} S_{n_{k_{j}}} \rightarrow \int_{0}^{1} w_{n} f$. But once again

$$
\int_{0}^{1} w_{n} S_{n_{k_{j}}}=\left\{\begin{array}{l}
0 \text { if } n_{k_{j}}<n \\
a_{n} \text { otherwise }
\end{array} .\right.
$$

Therefore $\hat{f}_{n}=\int_{0}^{1} w_{n} f=a_{n}$ for all $n$.

In order to see that $f \in L_{\Phi}[0,1]$, use Szlenk's theorem (see [9]) to extract yet another subsequence $\left\langle R_{j}\right\rangle_{j \in \mathbf{N}}$ of $\left\langle S_{n_{k_{j}}}\right\rangle_{j \in \mathbf{N}}$ with arithmetic means converging to $f$ in $L_{1}$-norm (that is, $\left\|f-\frac{1}{m} \sum_{j=1}^{m} R_{j}\right\|_{1} \rightarrow 0$ as $m \rightarrow \infty)^{1}$. Now choose a subsequence $\left\langle m_{k}\right\rangle$ of the positive integers so that $\frac{1}{m_{k}} \sum_{j=1}^{m_{k}} R_{j} \rightarrow f$ almost surely. Then $\Phi\left(\frac{1}{m_{k}} \sum_{j=1}^{m_{k}} \frac{R_{j}}{M}\right) \rightarrow \Phi\left(\frac{f}{M}\right)$ almost surely and so the convexity of $\Phi$ in tandem with Fatou's lemma yields

$$
\int_{0}^{1} \Phi\left(\frac{f}{M}\right) \leq \liminf _{k \rightarrow \infty} \int_{0}^{1} \Phi\left(\frac{1}{m_{k}} \sum_{j=1}^{m_{k}} \frac{R_{j}}{M}\right) \leq \sup _{k \in \mathrm{N}} \frac{1}{m_{k}} \sum_{j=1}^{m_{k}} \int_{0}^{1} \Phi\left(\frac{R_{j}}{M}\right) \leq 1 .
$$

Thus $f \in L_{\Phi}[0,1]$ and $\|f\|_{(\Phi)} \leq M$.

Corollary 3.2 Suppose that $\Phi \in \Delta_{2}$. The following are equivalent for a general Walsh series $S=\left\langle S_{n}\right\rangle_{n \in \mathbf{N}}=$ $\sum_{n=0}^{\infty} a_{n} w_{n}$ :

\footnotetext{
${ }^{1}$ An more direct approach could involve the deep theorem of J. Komlós (see [5]) which states that every $L_{1}$-bounded sequence admits a subsequence, each subsequence of which converges almost surely to the same function $f \in L_{1}[0,1]$.
} 
1. There is an $f \in L_{\Phi}[0,1]$ so that $a_{n}=\hat{f}_{n}$ for all $n$. That is, $S=S f$ for some $f \in L_{\Phi}[0,1]$.

2. There is subsequence $\left\langle S_{n_{k}}\right\rangle_{k \in \mathbf{N}}$ of $\left\langle S_{n}\right\rangle_{n \in \mathbf{N}}$ so that $\left\langle\Phi\left(S_{n_{k}}\right)\right\rangle_{k \in \mathbf{N}}$ is uniformly integrable.

3. There is subsequence $\left\langle S_{n_{k}}\right\rangle_{k \in \mathbf{N}}$ of $\left\langle S_{n}\right\rangle_{n \in \mathbf{N}}$ so that $\left\langle S_{n_{k}}\right\rangle_{k \in \mathbf{N}}$ has equi-absolutely continuous $L_{\Phi}$-norms.

4. There is a subsequence $\left\langle S_{n_{k}}\right\rangle_{k \in \mathbf{N}}$ of $\left\langle S_{n}\right\rangle_{n \in \mathbf{N}}$ so that $\left\langle\Phi\left(S_{n_{k}}\right)\right\rangle_{k \in \mathbf{N}}$ is $L_{1}$-bounded.

5. There is a subsequence $\left\langle S_{n_{k}}\right\rangle_{k \in \mathbf{N}}$ of $\left\langle S_{n}\right\rangle_{n \in \mathbf{N}}$ so that $\left\langle S_{n_{k}}\right\rangle_{k \in \mathbf{N}}$ is $L_{\Phi}$-bounded.

Proof: $\quad(1 \Rightarrow 2)$ : Suppose there is an $f \in L_{\Phi}[0,1]$ so that $a_{n}=\hat{f}_{n}$ for all $n$. Since $\Phi \in \Delta_{2}$ we have $\Phi(f) \in L_{1}[0,1]$ and by the conditional Jensen inequality

$$
\Phi\left(S_{2^{k}} f\right)=\Phi\left(\mathbf{E}\left(f \mid \mathbf{A}_{k}\right)\right) \leq \mathbf{E}\left(\Phi(f) \mid \mathbf{A}_{k}\right)=S_{2^{k}} \Phi(f)
$$

and since $\Phi(f) \in L_{1}[0,1]$, Theorem 2.1 ensures that $\left\langle S_{2^{k}} \Phi(f)\right\rangle_{k \in \mathbf{N}}$ and thus $\left\langle\Phi\left(S_{2^{k}} f\right)\right\rangle_{k \in \mathbf{N}}$ are uniformly integrable.

$(2 \Rightarrow 3)$ : Suppose that there is subsequence $\left\langle S_{n_{k}}\right\rangle_{k \in \mathbf{N}}$ of $\left\langle S_{n}\right\rangle_{n \in \mathbf{N}}$ so that $\left\langle\Phi\left(S_{n_{k}}\right)\right\rangle_{k \in \mathbf{N}}$ is uniformly integrable. Since $\Phi \in \Delta_{2}$, by 1.2 note 8(c) (also see [1, lemma 2.1]), $\left\langle S_{n_{k}}\right\rangle_{k \in \mathbf{N}}$ has equi-absolutely continuous $L_{\Phi}$-norms.

$(3 \Rightarrow 4)$ : Trivial

$(4 \Rightarrow 5)$ : Suppose that there is a subsequence $\left\langle S_{n_{k}}\right\rangle_{k \in \mathbf{N}}$ of $\left\langle S_{n}\right\rangle_{n \in \mathbf{N}}$ so that $\sup _{k \in \mathbf{N}} \int_{0}^{1} \Phi\left(S_{n_{k}}\right) \leq C$ for some $\dot{C}>0$. Let $\Psi$ denote the complement of $\Phi$. Then for each $k$, Young's inequality yields

$$
\left\|S_{n_{k}}\right\|_{\Phi}=\sup \left\{\left|\int_{0}^{1} g S_{n_{k}}\right|: \int_{0}^{1} \Psi(g) \leq 1\right\} \leq \int_{0}^{1} \Phi\left(S_{n_{k}}\right)+\sup \left\{\left|\int_{0}^{1} \Psi(g)\right|: \int_{0}^{1} \Psi(g) \leq 1\right\} \leq C+1 .
$$

Therefore $\sup _{k \in \mathbf{N}}\left\|S_{n_{k}}\right\|_{\Phi}<\infty$.

$(5 \Rightarrow 1)$ : This follows directly from theorem 3.1 .

The theorem of De La Vallée Poussin together with Theorem 3.1 and Corollary 3.2 gives several equivalent criteria which determine when a general Walsh series is the Walsh-Fourier series of a function in $L_{p}[0,1]$ for $1 \leq p<\infty$. The next two corollaries summarize some of them:

Corollary 3.3 The following are equivalent for a general Walsh series $S=\left\langle S_{n}\right\rangle_{n \in \mathbf{N}}=\sum_{n=0}^{\infty} a_{n} w_{n}$ :

1. $\left\langle S_{n}\right\rangle_{n \in \mathbf{N}}=\sum_{n=0}^{\infty} a_{n} w_{n}$ is the Walsh-Fourier series of an $f \in L_{1}[0,1]$.

2. There is an $N$-function $\Phi \in \Delta_{2}$ so that $\left\langle S_{n}\right\rangle_{n \in \mathbf{N}}=\sum_{n=0}^{\infty} a_{n} w_{n}$ is the Walsh-Fourier series of an $f \in L_{\Phi}[0,1]$.

3. There is an $N$-function $\Phi$ so that $\left\langle S_{n}\right\rangle_{n \in \mathbf{N}}=\sum_{n=0}^{\infty} a_{n} w_{n}$ is the Walsh-Fourier series of an $f \in L_{\Phi}[0,1]$. 
Proof: $(1 \Rightarrow 2)$ : By theorem 2.1 there is a uniformly integrable subsequence $\left\langle S_{n_{k}}\right\rangle_{k \in \mathbf{N}}$ of $\left\langle S_{n}\right\rangle_{n \in \mathbf{N}}$ and thus by Alexopoulos' improvement to the classical De La Vallée Poussin theorem (see 1.2 note 8b and [1]), there is an $N$-function $\Phi \in \Delta_{2}$ so that $\left\langle S_{n_{k}}\right\rangle_{k \in \mathbf{N}}$ has equi-absolutely continuous $L_{\Phi}$-norms. Corollary 3.2 finishes the job.

$(2 \Rightarrow 3)$ : Trivial

$(3 \Rightarrow 1)$ : Trivial since $L_{\Phi}[0,1] \subset L_{1}[0,1]$ for any $N$-function $\Phi$.

Corollary 3.4 The following are equivalent for a general Walsh series $S=\left\langle S_{n}\right\rangle_{n \in \mathbf{N}}=\sum_{n=0}^{\infty} a_{n} w_{n}$ :

1. $S=\left\langle S_{n}\right\rangle_{n \in \mathbf{N}}=\sum_{n=0}^{\infty} a_{n} w_{n}$ is the Walsh-Fourier series of an $f \in L_{\Phi}[0,1]$ for some $N$-function $\Phi$ whose complement $\Psi$ satisfies the $\Delta_{2}$ condition.

2. $S=S f$ for some function $f \in L_{p}[0,1]$ where $1<p<\infty$.

Proof: $\quad(1 \Rightarrow 2)$ : If $\Psi$ satisfies the $\Delta_{2}$ condition then there is a constants $K>0$ and $q>1$ so that $\Psi(x) \leq K|x|^{q}$ for large values of $x$. Thus there is a constant $C>0$ so that $C|x|^{p} \leq \Phi(x)\left(\frac{1}{p}+\frac{1}{q}=1\right)$ for large values of $x$. Hence the implication follows from the inclusion $L_{\Phi} \subseteq L_{p}$.

$(2 \Rightarrow 1)$ : The complement $\Psi$ of the $N$-function $\Phi$ defined by $\Phi(x)=\frac{|x|^{p}}{p}$ is given by $\Psi(x)=\frac{|x|^{q}}{q}$ where $\frac{1}{p}+\frac{1}{q}=1$. Plainly $\Psi \in \Delta_{2}$.

Now we will investigate the behaviour of Walsh series in $L_{\Phi}[0,1]$ for $\Phi \notin \Delta_{2}$ :

First, note that $\left\langle S_{n} f\right\rangle_{n \in \mathbf{N}} \subseteq E_{\Phi} \forall f \in L_{\Phi}$ and $E_{\Phi}$ is a closed subspace of $L_{\Phi}$. So if $f \in L_{\Phi} \backslash E_{\Phi}$ then no subsequence of $\left\langle S_{n} f\right\rangle_{n \in \mathbf{N}}$ converges to $f$ in $L_{\Phi}$ norm. In fact, much more is true:

Lemma 3.5 If the Walsh-Fourier series $\left\langle S_{n} f\right\rangle_{n \in \mathbf{N}}=\sum_{n=0}^{\infty} \hat{f}_{n} w_{n}$ of a function $f \in L_{\Phi}[0,1]$ has a subsequence $\left\langle S_{n_{k}} f\right\rangle_{k \in \mathbf{N}}$ that is weakly convergent to a function $g \in L_{\Phi}[0,1]$ then $f=g$ almost surely and thus $f \in E_{\Phi}$.

Proof: Suppose that $S_{n_{k}} f \stackrel{\text { weakly }}{\rightarrow} g$ as $k \rightarrow \infty$. Since $\left\langle S_{n} f\right\rangle_{n \in \mathbf{N}} \subseteq E_{\Phi}$ and $E_{\Phi}$ is a closed subspace of $L_{\Phi}$ we conclude that $g \in E_{\Phi}$. Now for each $n, w_{n} \in L_{\infty}[0,1] \subseteq E_{\Phi}^{*}=L_{\Psi}[0,1]$ (where $\Psi$ is the complement of $\Phi)$ and so

$$
\int_{0}^{1}\left(S_{n_{k}} f\right) w_{n} \rightarrow \int_{0}^{1} g w_{n} \text { as } k \rightarrow \infty .
$$

By the orthonormality of the Walsh functions $\int_{0}^{1} S_{n_{k}} w_{n}=\hat{f}_{n}$ for sufficiently large values of $k$ and so $\hat{f}_{n}=\int_{0}^{1} g w_{n}$. Hence $\int_{0}^{1} f w_{n}=\int_{0}^{1} g w_{n}$ or equivalently $\int_{0}^{1}(f-g) w_{n}=0, \forall n \in \mathbf{N}$. Since $f$ and $g$ are both in $L_{1}[0,1], f-g \in L_{1}[0,1]$ and so $S_{2^{n}}(f-g)=\sum_{k=0}^{2^{n}-1}(\widehat{f-g})_{k} w_{k}(x) \rightarrow f-g$ almost surely as $n \rightarrow \infty$. But $(\widehat{f-g})_{k}=\int_{0}^{1}(f-g) w_{k}=0, \forall k \in \mathbf{N}$. Therefore $f-g=0$ almost surely.

Corollary 3.6 If $f \in L_{\Phi} \backslash E_{\Phi}$ then no subsequence of $\left\langle S_{n} f\right\rangle_{n \in \mathbf{N}}$ possesses equi-absolutely continuous $L_{\Phi}$ norms. 
Proof: If $\left\langle S_{n_{k}}\right\rangle_{k \in \mathbf{N}}$ is a subsequence of $\left\langle S_{n} f\right\rangle_{n \in \mathbf{N}}$ with equi-absolutely continuous $L_{\Phi}$-norms then $\left\langle S_{n_{k}}\right\rangle_{k \in \mathbf{N}}$ has a weakly convergent subsequence (see 1.2 note $8(\mathrm{a})$ and [1, thm 2.3]) and thus by lemma $3.5 f \in E_{\Phi}$, contradicting the hypothesis.

\section{References}

[1] J. Alexopoulos, De La Vallée Poussin's theorem and weakly compact sets in Orlicz spaces, QM (1994), no. $17,231-248$.

[2] K. L. Chung, A course in probability theory, second ed., Academic Press, 1974.

[3] J. Diestel, Sequences and series in Banach spaces, Springer Verlag, 1984.

[4] N. Dunford and J. Schwartz, Linear operators part I: General theory, Wiley Classics library edition, Wiley Interscience, 1988.

[5] J. Komlós, A generalization of a problem of Steinhaus, Acta Mathematica Academiae Scientiarum Hungaricae (1967), no. 18, 217-229.

[6] M. A. Krasnoselskii and Ya. B. Rutickii, Convex functions and Orlicz spaces, Noorhoff Ltd., Groningen, 1961.

[7] P. Meyer, Probability and potentials, Blaisdell Publishing Co., 1966.

[8] F. Schipp, W.R. Wade and P. Simon, Walsh series: an introduction to dyadic harmonic analysis, Adam Hilger, Bristol and New York, 1990.

[9] W. Szlenk, Sur les suites faiblements convergentes dans l'espace L, Studia Math. (1965), no. 25, 337-341.

[10] A. Zygmund, Trigonometric series, second ed., Cambridge University Press, Cambridge, 1990.

John Alexopoulos, jalexopoulos@stark.kent.edu

Department of Mathematics, The Banach Center

Kent State University, Stark Campus

6000 Frank Avenue N.W.

Canton, OH 44720
Emily Sprague, esprague@mcs.kent.edu

Department of Mathematics

Kent State University

Kent, OH 44242 